\title{
NATURAL EXTENSIONS FOR PIECEWISE AFFINE MAPS VIA HOFBAUER TOWERS
}

\author{
HENK BRUIN AND CHARLENE KALLE
}

\begin{abstract}
We use canonical Markov extensions (Hofbauer towers) to give an explicit construction of the natural extensions of various measure preserving endomorphisms, and present some applications to particular examples.
\end{abstract}

\section{INTRODUCTION}

A measure theoretical dynamical system is a quadruple $(X, \mathcal{B}, \mu, T)$, where $(X, \mathcal{B}, \mu)$ is a probability space and $T: X \rightarrow X$ is a transformation that preserves the measure $\mu$, i.e., $\mu\left(T^{-1} A\right)=\mu(A)$ for each set $A \in \mathcal{B}$. To study the properties of a non-invertible transformation $T$, one can use a natural extension. This is a bigger, invertible system $(Y, \mathcal{C}, \nu, S)$ that preserves both the original dynamics and the measure structure with $\mathcal{C}$ being the coarsest $\sigma$-algebra that makes this possible. Many properties of a natural extension carry over to the original system. For example, the measure theoretical entropies of both systems are equal and they have the same mixing properties. In [Roh64], Rohlin gave a canonical construction of a natural extension for a wide class of dynamical systems on Lebesgue spaces. He showed that any two natural extensions of the same system are isomorphic, hence we can speak of the natural extension. Different versions however can have their own advantages.

As a basic example, consider the angle doubling map $T_{2} x=2 x(\bmod 1)$ on the unit interval $[0,1)$, preserving Lebesgue measure. A geometric version of the natural extension is given by the Baker transformation (Figure 1):

$$
B:[0,1)^{2} \rightarrow[0,1)^{2}, \quad(x, y) \mapsto(2 x(\bmod 1),(y+\lfloor 2 x\rfloor) / 2) .
$$

Here the dimension of the space $Y$ is one larger than the dimension of $X$, giving room to separate preimage branches. We can recover the original system simply by projecting onto the first coordinate.

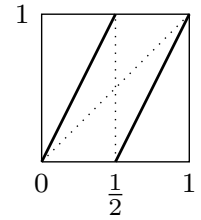

(a) The map $T_{2}$
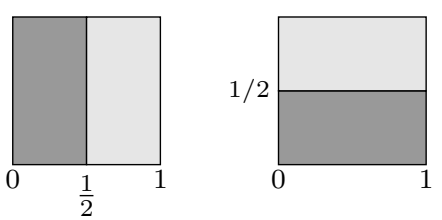

(b) The Baker transformation $B$ maps areas on the left to areas on the right with the same colour.

FiguRE 1. The angle doubling map and a geometric version of its natural extension.

Date: Version of September 30, 2018.

2000 Mathematics Subject Classification. Primary 37E05 Secondary 58F11, 28D05, 37A05.

Key words and phrases. Natural extension, Piecewise affine maps, Hofbauer tower.

The second author was supported by the research grant FWF S6913 and the NWO Veni grant 693.031.140. The research was partly supported by the EU FP6 Marie Curie Research Training Network CODY (MRTN 2006 035651). 
Such a geometric version of the natural extension can be used for various purposes. For example, for $\beta$-transformations (i.e., $x \mapsto \beta x(\bmod 1))$ it yields an explicit expression of the density of the absolutely continuous invariant measure, see [DKS96, DK09. In case $\beta$ is a Pisot number, certain geometric representations of algebraic natural extensions serve to identify periodic points (see for example [Aki02, IR06]) and are associated to multiple tilings of a Euclidean space (see for example [KS10, Sch00]). For the standard continued fraction transformation $x \mapsto \frac{1}{x}-\left\lfloor\frac{1}{x}\right\rfloor$, also called the Gauss map, a geometric natural extension substantially simplified proofs of results on the quality of the continued fraction approximation coefficients, such as the Doeblin-Lenstra Conjecture and generalisations of Borel's Theorem (see [Jag86, JK89]). For the $\alpha$-continued fraction map $T_{\alpha}:[\alpha-1, \alpha) \rightarrow[\alpha-1, \alpha), x \mapsto\left|\frac{1}{x}\right|-\left\lfloor\left|\frac{1}{x}\right|+1-\alpha\right\rfloor$ for parameter $\alpha \in[0,1]$ a geometric version was recently used to study the behaviour of the entropy as a function of $\alpha$ in KSS10.

In this paper we present a general method for obtaining geometric natural extensions of piecewise continuous maps with locally constant Jacobian $J(x)=\frac{d \mu \circ T}{d \mu}(x)$, which generalises the piecewise linearity of some of the above examples. The construction is based on the "canonical Markov extension" approach introduced by Hofbauer in [Hof80], commonly called Hofbauer tower; see also investigations by Buzzi [Buz99] and Bruin [Bru95]. In short, we apply the above "extend the space with one dimension" approach that works for the doubling map to the Hofbauer tower. This can be found in Section 2. In Section 3 we show that this natural extension is isomorphic to a countable state Markov shift and that it has several induced transformations that are Bernoulli. In the last two sections we give examples of systems to which the construction applies. These include all piecewise linear expanding interval maps with positive entropy. Other examples are certain higher dimensional piecewise affine maps, in particular a specific skew-product transformation called the random $\beta$-transformation, and rational maps on their Julia set.

\section{The Construction}

In this section we give the construction of the Hofbauer tower and of the geometric natural extension for the class of maps we consider in this article. We first describe this class of transformations.

2.1. The class of transformations. Let $X$ be a compact subset of $\mathbb{R}^{n}, \mathcal{B}$ the Lebesgue $\sigma$ algebra on $X$ and $\mu$ a probability measure on $(X, \mathcal{B})$. Let $\mathcal{Z}=\left\{Z_{j}\right\}_{1 \leq j \leq N}$ be a collection of closed sets giving a partition of $X$, so $\mu\left(Z_{j}\right)>0$ for all $j, \mu\left(Z_{i} \cap Z_{j}\right)=0$ for $i \neq j$ and $\mu\left(\cup_{1 \leq j \leq N} Z_{j}\right)=1$. Let $T: X \rightarrow X$ satisfy the following conditions.

(c1) For each $Z \in \mathcal{Z}$, the map $T$ can be extended uniquely to a continuous injective map $T_{Z}: Z \rightarrow \overline{T(\text { int } Z)}$, where int $Z$ denotes the interior of $Z$ and the bar denotes the closure.

(c2) For each set $A \in \mathcal{B}$, also $T A, T^{-1} A \in \mathcal{B}$ and if $\mu(A)=0$, then also $\mu\left(T^{-1} A\right)=0$.

(c3) The partition $\mathcal{Z}$ generates $\mathcal{B}$ in forward time. In other words, $\bigvee_{k \geq 0} T^{-k} \mathcal{Z}=\mathcal{B}$, where $\bigvee_{k \geq 0} T^{-k} \mathcal{Z}$ denotes the smallest $\sigma$-algebra containing all cylinder sets, i.e., the elements of common refinements $\mathcal{Z}_{n}:=\bigvee_{k=0}^{n} T^{-k} \mathcal{Z}$.

Thus we assume in (c2) that $\mu$ is non-singular w.r.t. $T$, but not yet that $\mu$ is $T$-invariant. This assumption will either be made later, or, starting from a reference measure $\mu^{b}$, our construction will produce a $T$-invariant measure $\mu \ll \mu^{b}$ for which a geometric natural extensions will be constructed. The important step is that we acquire a Markov measure for the Hofbauer tower, which we explain in the following section. 
2.2. The Hofbauer tower. Recall that $\mathcal{Z}_{n}=\bigvee_{k=0}^{n} T^{-k} \mathcal{Z}$ denotes the collection of $(n+1)$ cylinder sets $Z_{j_{0} \cdots j_{n}}$, defined by

$$
Z_{j_{0} \cdots j_{n}}=Z_{j_{0}} \cap T^{-1} Z_{j_{1}} \cap \cdots \cap T^{-n} Z_{j_{n}}
$$

whenever $\mu\left(Z_{j_{0} \cdots j_{n}}\right)>0$. Hence $\mathcal{Z}_{0}=\mathcal{Z}$. To obtain the Hofbauer tower we consider the $n$-th images under $T$ of the $(n+1)$-cylinder sets and order them in a convenient way. Indeed, consider the closures of the sets

$$
T^{n} Z_{j_{0} \cdots j_{n}} \quad \text { for } \quad Z_{j_{0} \cdots j_{n}} \in \mathcal{Z}_{n}, n \geq 0
$$

with the equivalence relation $\sim$ given by $T^{n} Z_{j_{0} \cdots j_{n}} \sim T^{m} Z_{i_{0} \cdots i_{m}}$ if the measure of the symmetric difference $\mu\left(T^{n} Z_{j_{0} \cdots j_{n}} \triangle T^{m} Z_{i_{0} \cdots i_{m}}\right)=0$. Let $\mathcal{D}$ denote the set of equivalence classes under this relation. We will occasionally abuse notation and consider the elements of $\mathcal{D}$ as subsets of $X$ instead of equivalence classes. Note that $T^{n} Z_{j_{0} \cdots j_{n}} \subseteq Z_{j_{n}}$.

Clearly $\mathcal{D}$ is finite or countably infinite, so we can take an ordered index set $\alpha \subseteq \mathbb{N}=$ $\{1,2,3, \ldots\}$ and write $\mathcal{D}=\left\{D_{u}: u \in \alpha\right\}$. It is convenient to set $D_{u}=Z_{u}$ for $u=1, \ldots, N$, so that the first $N$ elements of $\mathcal{D}$ are simply the elements of $\mathcal{Z}$, and we call $\hat{X}_{0}=\sqcup_{u=1}^{N} D_{u}$ the base of the Hofbauer tower. The full Hofbauer tower $\hat{X}$ (see [Hof80]) is the disjoint union of the elements of $\mathcal{D}$,

$$
\hat{X}=\bigsqcup_{n \geq 0} \bigsqcup_{j_{0} \cdots j_{n}} T^{n} Z_{j_{0} \cdots j_{n}} / \sim=\bigsqcup_{u \in \alpha} D_{u}
$$

Remark 1. There is a choice to define the levels $D_{u}$ as images $T\left(D_{v} \cap Z_{j}\right)$ as done by Hofbauer [Hof80] and Keller [Kel89] or as partition elements $T D_{v} \cap Z_{j}$ restricted to levels, as is done by Buzzi, e.g. Buz95. This difference has no profound effect on the outcome; however we follow Buzzi here, as it makes it easier to interpret the dynamics on $\hat{X}$ as a one-sided subshift of $\left(\alpha^{\mathbb{N}}, \sigma\right)$.

When it is important to specify which component a point $\hat{x}$ in the Hofbauer tower belongs to, we write $\hat{x}=(x, D)$ or $(x, u)$ when $D=D_{u}$. The canonical projection $\pi: \hat{X} \rightarrow X$, $\hat{x}=(x, D) \mapsto x$, maps the Hofbauer tower onto $X$. Note that $\hat{\mathcal{B}}:=\mathcal{D} \vee \pi^{-1}(\mathcal{B})$ is the Lebesgue $\sigma$-algebra on $\hat{X}$.

We extend the dynamics of $T$ to $\hat{X}$. Let $D=T^{n} Z_{j_{0} \cdots j_{n}} \in \mathcal{D}$. Then, for each $1 \leq j \leq N$ such that $\mu\left(Z_{j} \cap T D\right)>0$, also $D^{\prime}:=Z_{j} \cap T D=T^{n+1} Z_{j_{0} \cdots j_{n} j} \in \mathcal{D}$. Define $\hat{T}: \hat{X} \rightarrow \hat{X}$ by

$$
\hat{x}=(x, D) \mapsto\left(T x, Z_{j} \cap T D\right) \text { if } T x \in Z_{j},
$$

and write an arrow $D \rightarrow D^{\prime}$ if this happens. By construction, $\mathcal{D}$ is a Markov partition of $(\hat{X}, \hat{T})$, and $\pi \circ \hat{T}=T \circ \pi$. The arrow relation on $(\hat{X}, \hat{T})$ gives rise to a canonical Markov graph $(\mathcal{D}, \rightarrow)$. Define the symbol space

$$
\Sigma:=\left\{y=\left(y_{0} y_{1} y_{2} \ldots\right): y_{i} \in \alpha \text { and } D_{y_{i}} \rightarrow D_{y_{i+1}} \text { for all } i \geq 0\right\}
$$

indicating all the one-sided paths on $(\mathcal{D}, \rightarrow)$ and let $\sigma: \Sigma \rightarrow \Sigma$ denote the left shift, i.e., $(\sigma y)_{i}=y_{i+1}$. Let $\eta: \hat{X} \rightarrow \Sigma, \hat{x} \mapsto y$ be given by $y_{i}=u$ if $\hat{T}^{i} \hat{x} \in D_{u}$. The system $(\Sigma, \sigma)$ is a factor of $(\hat{X}, \hat{T})$ with factor map $\eta$, i.e., $\eta$ is surjective and $\eta \circ \hat{T}=\sigma \circ \eta$. A probability measure $\hat{\mu}$ on $(\mathcal{D}, \rightarrow)$ is called a Markov measure with transition probabilities $p_{u, v}$ if for all $u, v \in \alpha$

- $p_{u, v} \in[0,1], p_{u, v}=0$ when $D_{u} \nrightarrow \rightarrow D_{v}$ and $\sum_{v: D_{u} \rightarrow D_{v}} p_{u, v}=1$,

- $\sum_{u: D_{u} \rightarrow D_{v}} p_{u, v} \hat{\mu}\left(D_{u}\right)=\hat{\mu}\left(D_{v}\right)$.

We can extend the Markov measure $\hat{\mu}$ to $\mathcal{D} \vee \hat{T}^{-1} \mathcal{D}$ by defining $\hat{\mu}\left(D_{u} \cap \hat{T}^{-1} D_{v}\right)=p_{u, v} \hat{\mu}\left(D_{u}\right)$. Repeating this to cylinder sets of any length, and extending to the $\sigma$-algebra $\bigvee_{k \geq 0} \hat{T}^{-k} \mathcal{D}$, we automatically get that $\hat{\mu}$ is $\hat{T}$-invariant. We can take such Markov measures as starting point and define $\mu$ on $X$ as $\mu=\hat{\mu} \circ \pi^{-1}$. 
Lemma 1. For any Markov measure $\hat{\mu}$, the projected measure $\mu=\hat{\mu} \circ \pi^{-1}$ is T-invariant and satisfies conditions (c1)-(c3) of Section 2.1.

Proof. Conditions (c1), (c3) and the first part of (c2) do not mention a measure and are just part of the set-up. For the remaining part of condition (c2) we first show that $\bigvee_{k \geq 0} \hat{T}^{-k} \mathcal{D}=$ $\mathcal{D} \vee \pi^{-1}(\mathcal{B})$. Recall that $\mathcal{B}=\bigvee_{k \geq 0} T^{-k} \mathcal{Z}$. Hence,

$$
\mathcal{D} \vee \pi^{-1}(\mathcal{B})=\bigvee_{k \geq 0} \mathcal{D} \vee \pi^{-1}\left(T^{-k} \mathcal{Z}\right)
$$

To show that $\mathcal{D} \vee \pi^{-1}(\mathcal{B}) \subseteq \bigvee_{k \geq 0} \hat{T}^{-k} \mathcal{D}$, take any cylinder $Z_{j_{0} \cdots j_{n}}$ and suppose that $\hat{\mu}\left(\pi^{-1}\left(Z_{j_{0} \cdots j_{n}}\right) \cap\right.$ $D)>0$ for some $D \in \mathcal{D}$. Then there is a set $D^{\prime} \in \mathcal{D}$ such that $D^{\prime}=\hat{T}^{n}\left(\pi^{-1}\left(Z_{j_{0} \cdots j_{n}}\right) \cap D\right)$ and hence $\pi^{-1}\left(Z_{j_{0} \cdots j_{n}}\right) \cap D \in \bigvee_{k=0}^{n} \hat{T}^{-k} \mathcal{D}$. The inclusion then follows since $\mathcal{D} \vee \pi^{-1}(\mathcal{B})$ is the smallest $\sigma$-algebra containing all sets of the form $\pi^{-1}\left(Z_{j_{0} \cdots j_{n}}\right) \cap D$. For the other inclusion, take a non-empty set of the form $D_{u_{0}} \cap \hat{T}^{-1} D_{u_{1}} \cap \cdots \cap \hat{T}^{-k} D_{u_{k}}$. This means that there exists a cylinder set $Z=Z_{j_{0} \cdots j_{n} j_{n+1} \cdots j_{k}}$ such that $T^{n} Z \subseteq D_{u_{0}}$ and $T^{n+k} Z=D_{u_{k}}$. By the first part of (c2) $T^{n} Z \in \mathcal{B}$, so

$$
D_{u_{0}} \cap \hat{T}^{-1} D_{u_{1}} \cap \cdots \cap \hat{T}^{-k} D_{u_{k}}=D_{u_{0}} \cap \pi^{-1}\left(T^{n} Z\right) \in \mathcal{D} \vee \pi^{-1}(\mathcal{B}) .
$$

Hence, the two $\sigma$-algebras are equal. The $T$-invariance of $\mu$ then follows since $\hat{\mu}$ is $\hat{T}$-invariant and $T \circ \pi=\pi \circ \hat{T}$.

Example 1. One example, usually given for finite graphs, but valid for infinite graphs as well provided they are positive recurrent and hence the eigenvectors mentioned below belong to $\ell^{2}$ (see Gurevič [Gur69]), is the Parry measure, see [Wal82, Section 8.3]. To construct this measure, we assume for simplicity that the graph $(\mathcal{D}, \rightarrow)$ is primitive, and we let $A=\left(a_{t, u}\right)_{t, u \in \alpha}$ be its adjacency matrix given by $a_{t, u}=1$ if $D_{t} \rightarrow D_{u}$ and $a_{t, u}=0$ otherwise. Let $\lambda$ be the leading eigenvalue; by the Perron-Frobenius Theorem $\lambda>0$ and its associated left eigenvector $\bar{v}=\left(\bar{v}_{u}\right)_{u \in \alpha}$ and right eigenvector $\bar{w}=\left(\bar{w}_{u}\right)_{u \in \alpha}$ can be taken strictly positive. We can scale $\bar{v}$ and $\bar{w}$ such that $\sum_{u \in \alpha} \bar{v}_{u} \bar{w}_{u}=1$, and construct a stochastic matrix

$$
P=\left(p_{t, u}\right), \quad p_{t, u}=\frac{a_{t, u} \bar{v}_{u}}{\lambda \bar{v}_{t}} .
$$

Finally, the Markov measure $\hat{\mu}\left(D_{u}\right)=\bar{v}_{u} \bar{w}_{u}$ for all $u \in \alpha$, and in general,

$$
\hat{\mu}\left(\left\{\hat{x}: \hat{T}^{k}(\hat{x}) \in D_{u_{k}}, 0 \leq k<n\right\}\right)=\bar{v}_{u_{0}} \bar{w}_{u_{0}} p_{u_{0}, u_{1}} \cdots p_{u_{n-2}, u_{n-1}},
$$

is called the Parry measure. Extended to the $\sigma$-algebra generated by the cylinder sets $\bigvee_{k=0}^{n} \hat{T}^{-k} \mathcal{D}$, it becomes the measure of maximal entropy of $(\hat{X}, \hat{T})$, see [KH95, Chapter 4.4].

2.3. Lifting measures to the Hofbauer tower. The above shows that the Markov structure of the Hofbauer Tower always gives a measure on the tower. In general we often have a measure $\mu$ on $(X, \mathcal{B})$ that behaves nicely with respect to the map $T$. We would like to determine if there exists a $\hat{T}$-invariant measure $\hat{\mu}$ on $\hat{X}$ such that $\hat{\mu} \circ \pi^{-1}$ has some relation to $\mu$. Below we follow two strategies of constructing such a measure $\hat{\mu}$, one in case $\mu$ is $T$-invariant and one in case $\mu$ is not.

Assume that we have a system $(X, \mathcal{B}, \mu, T)$ satisfying $(\mathrm{c} 1),(\mathrm{c} 2)$ and $(\mathrm{c} 3)$. First extend the measure $\mu$ to a measure $\bar{\mu}$ on $\hat{X}$ by setting

$$
\bar{\mu}(A)=\sum_{D \in \mathcal{D}}(\mu \circ \pi)(A \cap D)
$$


for all $A \in \hat{\mathcal{B}}$. Note that $\bar{\mu}$ is not (necessarily) $\hat{T}$-invariant, and can in principle be infinite albeit $\sigma$-finite. Since we have assumed that $\mu(Z)>0$ for each cylinder $Z$, we have $\bar{\mu}(D)>0$ for all $D \in \mathcal{D}$. Define a sequence of Cesaro means $\left(\hat{\mu}_{n}\right)_{n \geq 1}$ on $\hat{X}$ by setting

$$
\hat{\mu}_{n}(A)=\frac{1}{n} \sum_{k=0}^{n-1} \bar{\mu}\left(\hat{T}^{-k} A \cap \hat{X}_{0}\right) .
$$

Here the intersection with base $\hat{X}_{0}$ guarantees that $\hat{\mu}_{n}$ are all probability measures. The measure $\mu$ is called liftable if the sequence $\left\{\hat{\mu}_{n}\right\}_{n \geq 1}$ from (3) converges in the vague topology (i.e., weak topology on compacta ${ }^{1}$ to a non-zero measure $\hat{\mu}$. Conditions under which measures are liftable are extensively studied, see for example [Kel89, BT07, Buz99, PSZ08]. The main point is that there can be no accumulation of mass on the boundaries of sets in the Hofbauer tower and mass cannot escape to infinity. Fix $D_{u} \in \mathcal{D}$ and let

$$
\partial_{n} D_{u}=\left\{Z \in \mathcal{Z}_{n}: 0<\mu\left(Z \cap D_{u}\right)<\mu(Z)\right\} .
$$

In words, $\partial_{n} D_{u}$ contains all $(n+1)$-cylinders $Z$ such that $Z$ and $D_{u}$ have a non-trivial intersection and the cylinder $Z$ is not completely contained in $D_{u}$. The capacity of the map $T$ is defined by

$$
\operatorname{cap}(T)=\limsup _{n \rightarrow \infty} \frac{1}{n} \log \sup _{u \in \alpha} \#\left(\partial_{n} D_{u}\right) .
$$

For the proof of Proposition 2 and for later use, define the sets

$$
B_{u, n}=\bigcup_{Z \in \partial_{n} D_{u}}\left(Z \cap D_{u}\right)
$$

We use the notation $\partial A$ for the usual boundary of a set $A \subset \mathbb{R}^{n}$. For the liftability of $\mu$ and the construction of the natural extension in the next section we need to make three additional assumptions on our system.

(c4) For each $1 \leq j \leq N$ there is a constant $s_{j} \geq 1$ such that for all measurable sets $A \subseteq Z_{j}$, $\mu(T A)=s_{j} \mu(A)$.

(c5) $\mu$ is ergodic, i.e., if $T^{-1} A=A$ for some $A \in \mathcal{B}$, then $\mu(A)=0$ or 1 .

(c6) $\mu\left(\cup_{n} T^{n}\left(\cup_{j=1}^{N} \partial Z_{j}\right)\right)=0$.

Remark 2. (i) Condition (c4) requires that the Jacobian of $T$ (see [Par69]) is locally constant; thus $J_{\mu, T}:=\frac{d \mu \circ T}{d \mu}$ has zero distortion.

(ii) In (c5) we assume ergodicity without insisting on $T$-invariance. Ergodicity of $\mu$ implies that each limit point of $\left\{\hat{\mu}_{n}\right\}_{n \geq 1}$ is either zero, or a probability measure.

The next proposition gives some first properties of limit points of the sequence in (3).

Proposition 1. Let $\hat{\mu}$ be a limit point of the sequence $\left\{\hat{\mu}_{n}\right\}$ defined in (3). Then $\hat{\mu}$ is $\hat{T}$-invariant and ergodic. Also, $\hat{\mu} \circ \pi^{-1}$ is ergodic.

Proof. The $\hat{T}$-invariance of $\hat{\mu}$ follows since it is a limit of Cesaro means. For ergodicity, let $\hat{U} \subseteq \hat{X}$ be a measurable set such that $\hat{T}^{-1} \hat{U}=\hat{U}$. Write $U=\pi(\hat{U})$. Then $T^{-1} U=U$, so by (c5) either $\mu(U)=0$ or $\mu(X \backslash U)=0$. If $\mu(U)=0$, then

$$
\bar{\mu}\left(\hat{T}^{-k}(\hat{U}) \cap \hat{X}_{0}\right)=(\mu \circ \pi)\left(\hat{T}^{-k}(\hat{U}) \cap \hat{X}_{0}\right) \leq(\mu \circ \pi)\left(\hat{T}^{-k} \hat{U}\right) \leq \mu\left(T^{-k} U\right)=0
$$

for each $k \geq 0$. Hence, $\hat{\mu}_{n}(\hat{U})=0$ for all $n$ and so $\hat{\mu}(\hat{U})=0$. Similarly, if $\mu(X \backslash U)=0$, then $\hat{\mu}(\hat{X} \backslash \hat{U})=0$. Hence $\hat{\mu}$ is ergodic.

For the last part, let $U \subseteq X$ be a measurable and $T$-invariant set. Then $\pi^{-1}\left(T^{-1} U\right)=$ $\hat{T}^{-1} \pi^{-1}(U)$ and by the previous, $(\hat{\mu} \circ \pi)(U)$ is either 0 or 1 .

\footnotetext{
${ }^{1}$ Recall that the space $X$ and also the levels $D$ are compact, so $\left(\left.\hat{\mu}_{n}\right|_{D}\right)_{n}$ has a weak accumulation point for each $D$
} 
Proposition 2 (Theorem 2 from $[$ Kel89]). Assume that $(X, \mathcal{B}, \mu, T)$ satisfies (c1)-(c6). For a T-invariant measure $\mu$ the sequence $\left\{\hat{\mu}_{n}\right\}_{n \geq 0}$ converges and if this limit $\hat{\mu} \not \equiv 0$, then $\hat{\mu}$ is an ergodic probability measure and $\hat{\mu} \circ \pi^{-1}=\mu$.

Proof. These results follow from Theorem 2 from [Kel89] by Keller, so we only need to check that the conditions of that theorem are satisfied: $T$ needs to be invariant and ergodic and there has to be a $\mu$-null set $N \subset X$ such that $\hat{N}=\pi^{-1} N$ has the properties

$(2.2) \pi^{-1}(A) \in \hat{\mathcal{B}}(\bmod \hat{N}) \Rightarrow A \in \mathcal{B}(\bmod \mu)$ for all $A \subseteq X$,

(2.3) $\hat{x}, \hat{y} \in \hat{X} \backslash \hat{N}$ and $\pi^{-1}(\hat{x})=\pi^{-1}(\hat{y})$ imply that $\exists n \geq 0$ s.t. $\hat{T}^{n} \hat{x}=\hat{T}^{n} \hat{y}$.

The ergodicity of $T$ is (c5) and the $T$-invariance is assumed in the proposition. Property (2.2) is satisfied since $\hat{\mathcal{B}}=\mathcal{D} \vee \pi^{-1}(\mathcal{B})$. Recall the definition of the sets $B_{u, n}$ from (6) and set $B_{u}=\cap_{n \geq 1} B_{u, n}$. Property (2.3) follows from (c6) when we take $N=\pi\left(\cup_{u \in \alpha} B_{u}\right)$, since this implies that the points $\hat{x}=\left(x, D_{u}\right)$ and $\hat{y}=\left(y, D_{v}\right)$ are not at the boundary of $D_{u}$ and $D_{v}$ respectively. Hence, there is some $n$ and some cylinder $Z_{n}$, such that $x, y \in Z_{n}$ and $Z_{n}$ is contained in the interior of $D_{u}$ and $D_{v}$ and this implies that $\hat{T}^{n} \hat{x}=\hat{T}^{n} \hat{y}$. This establishes the existence of a unique vague limit $\hat{\mu}$. If $\hat{\mu} \not \equiv 0$, then Theorem 2 from [Kel89] gives the rest of the statement: $\hat{\mu} \circ \pi^{-1}=\mu$ and $\hat{\mu}$ is ergodic.

Theorem 3 from [Kel89] by Keller gives conditions under which $\hat{\mu} \not \equiv 0$ in case of $T$-invariance.

Theorem 1 (Theorem 3, Kel89). Assume that $(X, \mathcal{B}, \mu, T)$ satisfies (c1)-(c6) and that $\mu$ is $T$-invariant. If $h_{\mu}(T)>\operatorname{cap}(T)$, where $h_{\mu}(T)$ denotes the metric entropy, then the sequence $\left\{\hat{\mu}_{n}\right\}_{n \geq 1}$ converges to an ergodic $\hat{T}$-invariant probability measure $\hat{\mu}$ for which $\hat{\mu} \circ \pi^{-1}=\mu$. Moreover, $h_{\hat{\mu}}(\hat{T})=h_{\mu}(T)$.

Proof. Note that $T$-invariance of $\mu$ implies condition (c2). The result by Keller is then valid under (c1), (c3), (c5) and (c6).

Invariance of $\mu$ is essential in Theorem 1 because otherwise $h_{\mu}(T)$ is undefined, and $\hat{\mu} \circ \pi^{-1}=\mu$ will fail. However, Theorem 1 has a version which applies to measures $\mu$ that are non-singular but not necessarily $T$-invariant, as long as (c2) holds. This is due to Keller [Kel90, Theorem 3(a)] for piecewise smooth interval maps, see also [MvS], and [BT07] for the setting of complex polynomials. We give one more example for piecewise affine and expanding maps in $\mathbb{R}^{q}$. However, it seems fair to say that proving liftability is not easier than proving the existence of an invariant measure equivalent to Lebesgue.

Proposition 3. Let $X \subset \mathbb{R}^{q}$ be compact and assume that $T: X \rightarrow X$ is piecewise affine and expanding w.r.t. a finite partition $\mathcal{Z}$ such that each $Z \in \mathcal{Z}$ is a polytope bounded by $(q-1)$ dimensional hyperplanes. Then Lebesgue measure $m^{q}$ lifts to the Hofbauer tower.

Proof. Tsujii [Tsu01] proved that piecewise affine expanding maps as above have an absolutely continuous invariant probability measure $\mu$ with bounded density $h=\frac{d \mu}{d x}$. Moreover, there are only finitely many Lebesgue ergodic components (only one if $T$ is transitive), so by passing to a component, we can assume that $q$-dimensional Lebesgue measure $m^{q}$ is ergodic.

In short, there is no need to use the Hofbauer tower approach to find $\mu$. We prove the liftability nonetheless, because it will assist us in creating the natural extension.

Let $\rho>1$ be the expansion factor: $d(T(x), T(y))>\rho d(x, y)$ (where $d$ stands for the Euclidean distance), whenever $x$ and $y$ belong to the same partition element $Z$. Let $S:=m^{q-1}(\partial \mathcal{Z})$ be the $(q-1)$-dimensional measure of the hyperplanes forming the partition $\mathcal{Z}$; this quantity is finite by the assumptions on $\mathcal{Z}$. For $\eta>0$ small, let $B(\eta)$ be an $\eta$-neighbourhood of $\partial \mathcal{Z}$ and let $\chi_{\eta}$ be the indicator function of $B(\eta)$. If $d(x, \partial \mathcal{Z})<\eta$, and $y \in \partial \mathcal{Z}$ is closest to $x$, then it takes at most 
$\left\lceil\frac{\log \eta-\log d_{\eta}(x, y)}{\log \rho}\right\rceil$ iterates to move $x$ and $y$ at least $\eta$ apart. Hence, for the first $n$ iterates in the orbit of $x$,

$$
\sum_{j=0}^{n-1} \chi_{\eta}\left(T^{j}(x)\right) \cdot \frac{\log \eta-\log d\left(T^{j}(x), \partial \mathcal{Z}\right)}{\log \rho},
$$

is an upper bound for the number of iterates $k$ that $T^{k} x$ is less than $\eta$ away from the image of $\partial \mathcal{Z}$ taken along the same branch $T^{k-j}$ as $T^{j} x$ at its previous close visit to $\partial \mathcal{Z}$. For the remaining iterates $k$, there is a neighbourhood $U_{k} \ni x$ such that $T^{k}$ maps $U_{k}$ homeomorphically (and in fact affinely) onto an $\eta$-ball around $T^{k} x$. In other words, $x$ has reached $\eta$-large scale at time $k$.

By the Ergodic Theorem, for $m^{q}$-a.e. $x$,

$$
\begin{aligned}
\frac{1}{n} \sum_{k=0}^{n-1} \chi_{\eta}\left(T^{k}(x)\right) & \frac{\log \eta-\log d\left(T^{k}(x), \partial \mathcal{Z}\right)}{\log \rho} \\
& \rightarrow \frac{1}{\log \rho} \int_{B(\eta)}(\log \eta-\log d(\xi, \partial \mathcal{Z})) d \mu(\xi) \\
& \leq \frac{2 S \sup h}{\log \rho} \int_{0}^{\eta}(\log \eta-\log \xi) d \xi=\frac{2 S \sup h}{\log \rho} \cdot \eta .
\end{aligned}
$$

Thus, the limit frequency that Lebesgue typical points reach $\eta$-large scale is $1-\frac{2 \eta S \sup h}{\log \rho} \ll 1$ for small $\eta$. When lifting the orbit of such typical $x$ to the Hofbauer tower, it will spend a similar proportion of time in a compact part $K$ of the tower, where $K$ depends only on $T$ and $\eta$. In probabilistic terms, the sequence $\left(\frac{1}{n} \sum_{k=0}^{n-1} \bar{m}^{q} \circ \hat{T}^{-k}\right)_{n}$ is tight, and this suffices to conclude that Lebesgue measure is liftable, say to $\hat{\mu}$. Naturally, $\hat{\mu} \circ \pi^{-1}=\mu$.

The next two examples show that expansion of Jacobian (rather than uniform expansion in all directions) or having positive Lyapunov exponents can both be insufficient for liftability.

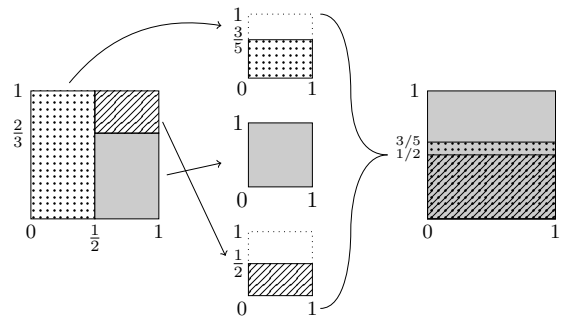

(a)

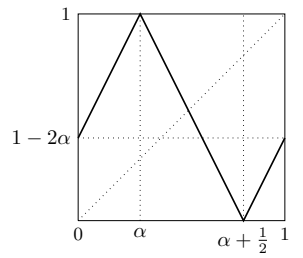

(b)

Figure 2. In (a) we see the map from Example 2, (b) shows the map from Example 3, which is a bimodal interval map with slope \pm 2 and turning points $\alpha$ and $\frac{1}{2}+\alpha$.

Example 2. Consider the skew product $T:[0,1) \times[0,1)$ defined as

$$
T(x, y)=(2 x(\bmod 1), a(x) y(\bmod 1)) \quad \text { for } \quad a(x)= \begin{cases}\frac{3}{5} & \text { if } x \in\left[0, \frac{1}{2}\right), \\ \frac{3}{2} & \text { if } x \in\left[\frac{1}{2}, 1\right),\end{cases}
$$

see Figure 2(a). This map is transitive and the Jacobian of $T$ w.r.t. Lebesgue measure $m^{2}$ is expanding and locally constant: $J_{m^{2}, T}(x)=2 a(x)$. Note that for the omega-limit set $\omega(x, y)=$ 
$\cap_{n \geq 0} \overline{\left\{T^{k}(x, y): k>n\right\}}$ we have $\omega(x, y)=[0,1) \times\{0\}$ for $m^{2}$-a.e. $(x, y)$; this is by a standard argument of skew-products because the Lebesgue typical transversal Lyapunov exponent is $\int \log a(x) d x=\log 9 / 10<0$. This implies that the unique weak limit measure of $\frac{1}{n} \sum_{k=0}^{n-1} m^{2} \circ$ $T^{-k}$ is one-dimensional Lebesgue measure on $[0,1) \times\{0\}$, i.e., Lebesgue measure $m^{2}$ is not liftable.

Example 3. Define the interval map $T:[0,1) \rightarrow[0,1)$ by

$$
T(x)= \begin{cases}2 x+1-2 \alpha & \text { if } x \in[0, \alpha), \\ 1+\alpha-2 x & \text { if } x \in\left[\alpha, \alpha+\frac{1}{2}\right), \\ 2 x-2 \alpha-1 & \text { if } x \in\left[\alpha+\frac{1}{2}, 1\right),\end{cases}
$$

see Figure 2(b). For certain values of $\alpha$, the set of points whose orbits stay in $[0, \alpha] \cup\left[\alpha+\frac{1}{2}, 1\right)$ form a Cantor set $H$ of zero Hausdorff dimension on which $T$ is semi-conjugate to a circle rotation. The measure obtained from lifting Lebesgue measure to $H$ is invariant (hence of Jacobian 1), has zero entropy but Lyapunov exponent $\log 2$. It is not liftable to the Hofbauer tower. This example was inspired by [HR89], see also [BT09].

2.4. Piecewise constant Radon-Nikodym derivatives. Assumption (c4) implies that if $D \rightarrow D^{\prime}$ and $D \subset Z_{j}$, then the Jacobian $J_{\bar{\mu}, \hat{T}}=s_{j}$ on $D$. The next lemma shows that the Radon-Nikodym derivative $\frac{d \hat{\mu}}{d \bar{\mu}}$ is constant on $D$ as well. We need this for the construction of the natural extension.

Lemma 2. If (c1)-(c4) hold for the system $(X, \mathcal{B}, \mu, T)$, then $\hat{\mu}_{n} \ll \bar{\mu}$ for each $n \geq 1$. Moreover, the densities $\frac{d \hat{\mu}_{n}}{d \bar{\mu}}$ are constant on each $D \in \mathcal{D}$.

Proof. Fix $D \in \mathcal{D}$ and take a measurable set $A \subseteq D$. Note that for each $(k+1)$-cylinder $Z_{j_{0} \cdots j_{k}} \in \mathcal{Z}_{k}$ with $T^{k} Z_{j_{0} \cdots j_{k}}=D$, by (c4) we have

$$
\left(s_{j_{0}} \cdots s_{j_{k-1}}\right) \mu\left(\pi\left(\hat{T}^{-k} A \cap \hat{X}_{0}\right) \cap Z_{j_{0} \cdots j_{k}}\right)=\mu(\pi(A))=\bar{\mu}(A),
$$

where an empty product $s_{j_{0}} \cdots s_{j_{k-1}}$ for $k=0$ is taken as 1 . Hence,

$$
\begin{gathered}
\bar{\mu}\left(\hat{T}^{-k} A \cap \hat{X}_{0}\right)=\sum_{\substack{Z_{j_{0} \cdots j_{k} \in \mathcal{Z}_{k}:} \\
T^{k} Z_{j_{0} \cdots j_{k}}=D}} \mu\left(\pi\left(\hat{T}^{-k} A \cap \hat{X}_{0}\right) \cap Z_{j_{0} \cdots j_{k}}\right) \\
=\sum_{\substack{Z_{j_{0} \cdots j_{k} \in \mathcal{Z}_{k}:}: \\
T^{k} Z_{j_{0} \cdots j_{k}}=D}} \frac{\bar{\mu}(A)}{s_{j_{0}} \cdots s_{j_{k-1}}} .
\end{gathered}
$$

Passing to the Cesaro mean, this implies that $\hat{\mu}_{n} \ll \bar{\mu}$. Also, we can write $\hat{\mu}_{n}(A)=\int_{A} \rho_{n}(D) d \bar{\mu}$ with

$$
\rho_{n}(D):=\frac{1}{n} \sum_{k=0}^{n-1} \sum_{\substack{Z_{j_{0} \cdots j_{k}}: \\ T^{k} Z_{j_{0} \cdots j_{k}}=D}}\left(s_{j_{0}} \cdots s_{j_{k-1}}\right)^{-1} .
$$

Since $\rho_{n}(D)$ only depends on $D$, we get the lemma with $\left.\frac{d \hat{\mu}_{n}}{d \bar{\mu}}\right|_{D}=\rho_{n}(D)$.

Proposition 4. Assume that (c1)-(c4) hold for $(X, \mathcal{B}, \mu, T)$, and that $\hat{\mu}$ is a non-zero vague limit point of $\left\{\hat{\mu}_{n}\right\}_{n \geq 1}$. Then $\hat{\mu} \ll \bar{\mu}$ and the density $\frac{d \hat{\mu}}{d \bar{\mu}}$ is constant on each set $D \in \mathcal{D}$ and given by $\rho(D):=\left.\frac{d \hat{\mu}}{d \bar{\mu}}\right|_{D}=\frac{\hat{\mu}(D)}{\bar{\mu}(D)}$.

Remark 3. Note that $\hat{\mu} \ll \bar{\mu}$ implies that $\hat{\mu} \circ \pi^{-1} \ll \mu$. The previous proposition doesn't use $T$-invariance of $\mu$. Since $\hat{\mu}$ is $\hat{T}$-invariant even if $\mu$ is not $T$-invariant, $\hat{\mu} \circ \pi^{-1} \ll \mu$ is $T$-invariant and in the sequel we produce a natural extension of $\left(X, \mathcal{B}, \hat{\mu} \circ \pi^{-1}, T\right)$. 
Proof. Fix $D \in \mathcal{D}$ and $A \subset D$ compact. By Lemma $2, \rho_{n}(D)$ is constant, and since $\hat{\mu}$ is a vague limit point of the sequence $\left\{\hat{\mu}_{n}\right\}_{n \geq 1}$ along some subsequence $\left\{n_{k}\right\}_{k \geq 1},\left\{\left.\hat{\mu}_{n_{k}}\right|_{A}\right\}_{k \geq 1}$ converges to $\left.\hat{\mu}\right|_{A}$ in the weak topology as $k \rightarrow \infty$. This means that $\rho_{n_{k}}(D)=\frac{\hat{\mu}_{n_{k}}(A)}{\bar{\mu}(A)}$ converges to a constant limit density $\rho(D)$. Clearly $\hat{\mu}(D)=\rho(D) \bar{\mu}(D)$, so $\rho(D):=\left.\frac{d \hat{\mu}}{d \bar{\mu}}\right|_{D}=\frac{\hat{\mu}(D)}{\bar{\mu}(D)}$ follows.

2.5. The natural extension. From the Hofbauer tower we will obtain a version of the natural extension of the transformation $T$. We start from a system $(X, \mathcal{B}, \mu, T)$ satisfying (c1)-(c6) and we assume that the measure $\mu$ is liftable, either by satisfying the requirements of Theorem 1 if $\mu$ is $T$-invariant or by other means (such as Proposition 3). Let us first give a formal definition of the natural extension.

Definition 1. A measure theoretical dynamical system $(Y, \mathcal{C}, \nu, F)$ is a natural extension of the non-invertible system $(X, \mathcal{B}, \mu, T)$ if all the following are satisfied. There are sets $X^{*} \in \mathcal{B}$ and $Y^{*} \in \mathcal{C}$, with $\mu\left(X^{*}\right)=1=\nu\left(Y^{*}\right)$ and $T\left(X^{*}\right) \subset X^{*}$ and $F\left(Y^{*}\right) \subseteq Y^{*}$ and there is a map $\phi: X^{*} \rightarrow Y^{*}$ such that

(ne1) $F$ is invertible $\nu$-a.e.;

(ne2) $\phi$ is bi-measurable and surjective;

(ne3) $\phi$ preserves the measure structure, i.e., $\mu=\nu \circ \phi^{-1}$;

(ne4) $\phi$ preserves the dynamics, i.e., $\phi \circ T=F \circ \phi$;

(ne5) $\mathcal{C}$ is the coarsest $\sigma$-algebra that makes (ne1)-(ne4) valid, i.e., $\bigvee_{n \geq 0} F^{-n}\left(\phi^{-1} \mathcal{B}\right)=\mathcal{C}$.

If a map $\phi$ satisfies (ne2), (ne3), (ne4) and is injective, then the systems $(Y, \mathcal{C}, \nu, F)$ and $(X, \mathcal{B}, \mu, T)$ are called isomorphic and $\phi$ is an isomorphism.

For the natural extension only the components $D_{u}$ of positive $\hat{\mu}$-measure are important, but w.l.o.g. we can assume that $\hat{\mu}\left(D_{u}\right)>0$ for all $u \in \alpha$. To define the natural extension domain $Y$, extend each $D_{u}$ by one dimension to a set $R_{u}=D_{u} \times\left[0, \rho\left(D_{u}\right)\right]$, which we will call the $r u g$ of $D_{u}$. Set $Y:=\bigsqcup_{u \in \alpha} R_{u}$ and let $\mathcal{C}$ denote the Borel $\sigma$-algebra on $Y$. Use $\nu$ to denote the product measure on $(Y, \mathcal{C})$ given by $\bar{\mu} \times m$ on each rug, where $m$ is the one-dimensional Lebesgue measure. Then by Proposition 4

$$
\nu(Y)=\sum_{u \in \alpha} \bar{\mu}\left(D_{u}\right) \rho\left(D_{u}\right)=\sum_{u \in \alpha} \hat{\mu}\left(D_{u}\right)=1 .
$$

Let $\hat{\pi}: Y \rightarrow \hat{X}$ be the projection onto the first coordinate. Then $\nu \circ \hat{\pi}^{-1}=\hat{\mu}$. We will extend the action of $\hat{T}$ to the vertical direction, obtaining a new map which we call $F: Y \rightarrow Y$. This is done piecewise as follows. For $z=(\hat{x}, y, u) \in R_{u}$ with $\pi(\hat{x}) \in Z_{j}$ and $\hat{T} \hat{x} \in D_{v}$, define

$$
F z=F(\hat{x}, y, u)=\left(\hat{T} \hat{x}, \frac{y}{s_{j}}+\sum_{1 \leq k \leq N} \sum_{\substack{t<u: \\ \pi^{-1}\left(Z_{k}\right) \supset D_{t} \rightarrow D_{v}}} \frac{\rho\left(D_{t}\right)}{s_{k}}, v\right) .
$$

In words, the parts of all the rugs $R_{t}$ that map to $R_{v}$ are squeezed in the vertical direction by a factor equal to the expansion in the 'horizontal' direction and are stacked on top of each other into the rug $R_{v}$ according to the order relation on $\mathcal{D}$, see Figure 3 . Hence, the image strips in $R_{v}$ are disjoint. By Proposition 4 the map $F$ is well-defined on a full measure subset of $Y$. Since the stretch in the horizontal direction and the squeeze in vertical direction are the same, $F$ preserves area $\nu$. The next lemma gives (ne1).

Lemma 3. The map $F$ is invertible $\nu$-a.e. 


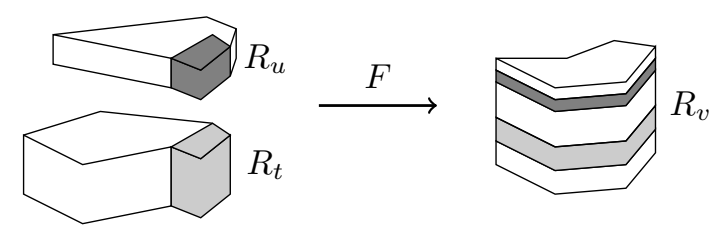

FiguRE 3. $F$ maps the coloured regions in $R_{t}$ and $R_{u}$ both to $R_{v}$, i.e., there is a set $Z_{j}$, such that $T D_{t} \cap Z_{j}=T D_{u} \cap Z_{j}=D_{v}$. If $t<u$, then the image of $R_{t}$ in $R_{v}$ lies below the image of $R_{u}$. Also, the image of $R_{t}$ and $R_{u}$ stretch all the way across $R_{v}$ in the "horizontal" direction.

Proof. To show that $F$ is surjective, first note that

$$
\begin{aligned}
\hat{\mu}\left(D_{u}\right) & =\hat{\mu}\left(\hat{T}^{-1} D_{u}\right)=\sum_{t \in \alpha} \int_{\hat{X}} \frac{d \hat{\mu}}{d \bar{\mu}} 1_{\hat{T}^{-1} D_{u} \cap D_{t}} d \bar{\mu} \\
& =\sum_{t \in \alpha} \rho\left(D_{t}\right) \bar{\mu}\left(D_{t} \cap \hat{T}^{-1} D_{u}\right) .
\end{aligned}
$$

Thus,

$$
\begin{aligned}
\rho\left(D_{u}\right) & =\frac{1}{\bar{\mu}\left(D_{u}\right)} \sum_{t \in \alpha} \rho\left(D_{t}\right) \bar{\mu}\left(D_{t} \cap \hat{T}^{-1} D_{u}\right) \\
& =\frac{1}{\bar{\mu}\left(D_{u}\right)} \sum_{1 \leq j \leq N} \sum_{\substack{t \in \alpha: \\
\pi^{-1}\left(Z_{j}\right) \supset D_{t} \rightarrow D_{u}}} \rho\left(D_{t}\right) \frac{\bar{\mu}\left(D_{u}\right)}{s_{j}}=\sum_{t \in \alpha} \sum_{\substack{1 \leq j \leq N: \\
\pi^{-1} Z_{j} \supset D_{t} \rightarrow D_{u}}} \frac{\rho\left(D_{t}\right)}{s_{j}} .
\end{aligned}
$$

This shows that every $(\hat{x}, y) \in R_{u}$ is the images of something; the $\hat{x}$-coordinate because $T D=D_{u}$ if $D \rightarrow D_{u}$, and the $y$-coordinate because only those $D_{t}$ with $D_{t} \rightarrow D_{u}$ contribute to the strips of the rug $R_{u}$.

For the injectivity of $F$, first note that for the horizontal boundary of each rug $R_{u}$ we have $\nu\left(D_{u} \times\left\{0, \rho\left(D_{u}\right)\right\}\right)=\bar{\mu}\left(D_{u}\right) \cdot 0=0$. Let $M$ be the union of all these boundaries, i.e., $M=$ $\cup_{u \in \alpha}\left(D_{u} \times\left\{0, \rho\left(D_{u}\right)\right\}\right)$. Then $\nu\left(\cup_{n \in \mathbb{Z}} F^{n} M\right)=0$. Assume that $\left(\hat{x}_{1}, y_{1}, t\right),\left(\hat{x}_{2}, y_{2}, u\right) \in Y \backslash M$ are such that

$$
F\left(\hat{x}_{1}, y_{1}, t\right)=F\left(\hat{x}_{2}, y_{2}, u\right)=(\hat{x}, y, v) .
$$

Then, by the injectivity of $T$ on each of the elements of $\mathcal{Z}=\left\{Z_{j}\right\}_{j=1}^{N}$, if $\hat{x}_{1} \neq \hat{x}_{2}$ with $\hat{x}_{1} \in D_{t} \subset$ $\pi^{-1}\left(Z_{j}\right)$ and $\hat{x}_{2} \in D_{u} \subset \pi^{-1}\left(Z_{k}\right)$, then either $t \neq u$ or $j \neq k$. By the definition of $F$, either one of these inequalities implies that the second coordinates of $F\left(\hat{x}_{1}, y_{1}, t\right)$ and $F\left(\hat{x}_{2}, y_{2}, u\right)$ cannot be equal. Hence $t=u$ and $\hat{x}_{1}=\hat{x}_{2}$. Since $F$ stacks the rugs on top of each other according to the ordering on $\mathcal{D}$, this implies that also $y_{1}=y_{2}$. Hence, $\left(\hat{x}_{1}, y_{1}, t\right)=\left(\hat{x}_{2}, y_{2}, u\right)$ and $F$ is invertible.

Note that $\hat{T} \circ \hat{\pi}=\hat{\pi} \circ F$, where as before, $\hat{\pi}: Y \rightarrow \hat{X}$ is the projection onto the first coordinate. Also, $\nu\left(\hat{\pi}^{-1}(B)\right)=\hat{\mu}(B)$ for all $B \in \hat{\mathcal{B}}$. Let $\phi:=\pi \circ \hat{\pi}: Y \rightarrow X$, see Figure 4. Since also $T \circ \pi=\pi \circ \hat{T}$ and $\hat{\mu} \circ \pi^{-1}=\mu, \phi$ satisfies (ne2), (ne3) and (ne4).

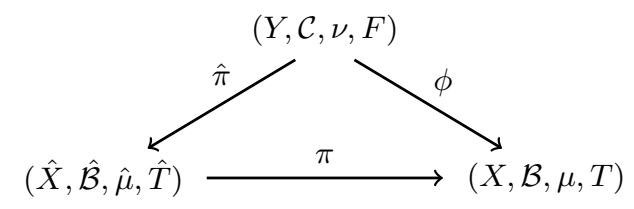

FiguRE 4. The three spaces involved and the projections between them. 
It remains to show (ne5). Recall the definition of the sets $\partial_{n} D_{u}$ from (4) and the sets $B_{u, n}$ from (6). Let $B_{n}=\bigsqcup_{u \in \alpha} B_{u, n}$ be the disjoint union of these sets over all $u$. Then $B_{n+1} \subseteq B_{n}$ and by (c6) and Proposition $4, \lim _{n \rightarrow \infty} \hat{\mu}\left(B_{n}\right)=0$. We extend the sets $B_{u, n}$ to $Y$ by defining

$$
E_{u, n}=\bigcup_{Z \in \mathcal{Z}_{n}}\left\{\left(Z \cap D_{u}\right) \times\left[0, \rho\left(D_{u}\right)\right]: 0<\bar{\mu}\left(Z \cap D_{u}\right)<\mu(Z)\right\} \subseteq Y .
$$

Also, let $E_{n}=\bigsqcup_{u \in \alpha} E_{u, n}$. Since $\hat{\mu}=\nu \circ \hat{\pi}^{-1}$, we have

$$
0 \leq \nu\left(\bigcap_{n \geq 0} E_{n}\right)=\lim _{n \rightarrow \infty} \nu\left(E_{n}\right) \leq \lim _{n \rightarrow \infty}\left(\nu \circ \hat{\pi}^{-1}\right)\left(B_{n}\right)=\lim _{n \rightarrow \infty} \hat{\mu}\left(B_{n}\right)=0 .
$$

We need the following lemma.

Lemma 4. If $z \in E_{n+1}$, then $F z \in E_{n}$ and hence $F^{n} E_{n+1} \subseteq F^{n-1} E_{n}$.

Proof. Let $z \in E_{n+1}$ and suppose $\hat{\pi}(z) \in Z_{j_{0} \cdots j_{n}} \cap D_{u}$ for some set $Z_{j_{0} \cdots j_{n}} \in \mathcal{Z}_{n+1}$ and $u \in \alpha$. Then there is a $v \in \alpha$, such that $\hat{T} D_{u}=D_{v} \subset \pi^{-1}\left(Z_{j_{1}}\right)$ and $\hat{\pi}(F z) \in \hat{T}\left(\pi^{-1}\left(Z_{j_{0} \cdots j_{n}}\right) \cap D_{u}\right)=$ $\pi^{-1}\left(Z_{j_{1} \cdots j_{n}}\right) \cap D_{v}$. Then,

$$
\begin{aligned}
0<\bar{\mu}\left(\pi^{-1}\left(Z_{j_{1} \cdots j_{n}}\right) \cap D_{v}\right) & =s_{j_{0}} \bar{\mu}\left(\pi^{-1}\left(Z_{j_{0} \cdots j_{n}}\right) \cap D_{u}\right) \\
& <s_{j_{0}} \mu\left(Z_{j_{0} \cdots j_{n}}\right) \leq \mu\left(Z_{j_{1} \cdots j_{n}}\right),
\end{aligned}
$$

which gives that $F z \in E_{n}$.

For points $z \in E_{n}$, the first $n$ iterates $F^{k} z$ lie close to the boundary of their rugs. We will prove (ne5) by showing that the map $F$ separates points. In order to make this work, we need to exclude points of which all inverse images lie close to the boundary.

Lemma 5. Under condition (c6) the $\sigma$-algebra $\bigvee_{n \geq 0} F^{n}\left(\phi^{-1} \mathcal{B}\right)$ is equal, up to a set of $\nu$-measure zero, to the $\sigma$-algebra $\mathcal{C}$ of Lebesgue measurable sets on $Y$.

Proof. First we define the exceptional set. Let $E=\bigcap_{n>1} F^{n-1} E_{n}$. Since $F$ is invertible almost everywhere, $\nu\left(F^{n-1} E_{n}\right)=\nu\left(E_{n}\right)$ for all $n \geq 1$. Lemma 4 implies that $F^{n} E_{n+1} \subseteq F^{n-1} E_{n}$ for all $n \geq 1$. Therefore

$$
\nu(E)=\lim _{n \rightarrow \infty} \nu\left(F^{n-1} E_{n}\right)=\lim _{n \rightarrow \infty} \nu\left(E_{n}\right)=0 .
$$

Let $z=(\hat{x}, y, u) \in R_{u} \backslash(F E)$ and $z^{\prime}=\left(\hat{x}^{\prime}, y^{\prime}, u^{\prime}\right) \in R_{u^{\prime}} \backslash(F E)$ be two different points in $Y$. It suffices to show that there are sets $B$ and $B^{\prime} \in \mathcal{B}$ and $n \geq 1$, such that $z \in F^{n} \phi^{-1}(B)$ and $z^{\prime} \in F^{n} \phi^{-1}\left(B^{\prime}\right)$ and moreover $F^{n} \phi^{-1}(B) \cap F^{n} \phi^{-1}\left(B^{\prime}\right)=\emptyset$.

Note that if $x=\phi(z) \neq x^{\prime}=\phi\left(z^{\prime}\right)$, then there are two disjoint open sets $B, B^{\prime} \subset X$, such that $x \in B$ and $x^{\prime} \in B^{\prime}$. Then $\phi^{-1}(B)$ and $\phi^{-1}\left(B^{\prime}\right)$ are still disjoint and contain $z$ and $z^{\prime}$ respectively.

Now suppose that $\phi(z)=\phi\left(z^{\prime}\right)$, but $u \neq u^{\prime}$. We introduce some notation. For $n \geq 1$, write $F^{-n} z \in R_{u_{n}}$ and use $Z^{\left(u_{n}\right)}$ to denote the $(n+1)$-cylinder in which $\phi\left(F^{-n} \hat{z}\right)$ lies. For $z^{\prime}$, we use $R_{u_{n}^{\prime}}$ and $Z^{\left(u_{n}^{\prime}\right)}$ respectively. Suppose that $\phi\left(F^{-n} z\right)=\phi\left(F^{-n} z^{\prime}\right)$ for all $n \geq 1$, i.e., $Z^{\left(u_{n}\right)}=Z^{\left(u_{n}^{\prime}\right)}$ for all $n \geq 1$. Since $z, z^{\prime} \notin E$, there are $k, k^{\prime} \geq 1$, such that $z \notin F^{k-1} E_{k}$ and $z^{\prime} \notin F^{k^{\prime}-1} E_{k^{\prime}}$. By Lemma 4 we have for all $n \geq \max \left\{k, k^{\prime}\right\}$, that $F^{-n+1} z, F^{-n+1} z^{\prime} \notin E_{n}$. This implies that

$$
\bar{\mu}\left(\pi^{-1}\left(Z^{\left(u_{n}\right)}\right) \cap D_{u_{n}}\right)=\mu\left(Z^{\left(u_{n}\right)}\right) \quad \text { and } \quad \bar{\mu}\left(\pi^{-1}\left(Z^{\left(u_{n}^{\prime}\right)}\right) \cap D_{u_{n}^{\prime}}\right)=\mu\left(Z^{\left(u_{n}^{\prime}\right)}\right)
$$

and that $Z^{\left(u_{n}\right)}=Z^{\left(u_{n}^{\prime}\right)}$. Thus,

$$
D_{u}=T^{n}\left(Z^{\left(u_{n}\right)} \cap D_{u_{n}}\right)=T^{n}\left(Z^{\left(u_{n}^{\prime}\right)} \cap D_{u_{n}^{\prime}}\right)=D_{u^{\prime}},
$$

a contradiction. Hence, there is an $n$ such that $\phi\left(F^{-n} z\right) \neq \phi\left(F^{-n} z^{\prime}\right)$. This means that we can find two disjoint open sets $B, B^{\prime} \subset X$, such that $\phi\left(F^{-n} z\right) \in B$ and $\phi\left(F^{-n} z^{\prime}\right) \in B^{\prime}$. Then again $F^{n} \phi^{-1}(B)$ and $F^{n} \phi^{-1}\left(B^{\prime}\right)$ are still disjoint and contain $z$ and $z^{\prime}$ respectively. 
Finally, if $z$ and $z^{\prime}$ are in the same rug with $y \neq y^{\prime}$, then, since $F$ is contracting in the vertical direction, there is an $n$, such that $F^{-n} z$ and $F^{-n} z^{\prime}$ are in different rugs and we can repeat the argument from above.

This lemma finishes the proof of the following theorem.

Theorem 2. Let $(X, \mathcal{B}, \mu, T)$ be a system that satisfies conditions (c1)-(c6) and assume $\mu$ lifts to a probability measure $\hat{\mu}$ on $(\hat{X}, \hat{\mathcal{B}})$. Then the system $(Y, \mathcal{C}, \nu, F)$ is the natural extension of $\left(X, \mathcal{B}, \hat{\mu} \circ \pi^{-1}, T\right)$ with factor map $\phi=\pi \circ \hat{\pi}$. In case $\mu$ is $T$-invariant, then $\hat{\mu}$ is the unique lift and $\hat{\mu} \circ \pi^{-1}=\mu$.

Corollary 1. Let $(X, \mathcal{B}, \mu, T)$ be a system that satisfies conditions (c1)-(c6). If $\mu$ is T-invariant and $h_{\mu}(T)>\operatorname{cap}(T)$, then $\nu$ is ergodic and $h_{\nu}(F)=h_{\mu}(T)$.

Proof. If $\mu$ is $T$-invariant, then $\hat{\mu} \circ \pi^{-1}=\mu$. By (c5) $\mu$ is ergodic and since ergodicity and metric entropy are preserved under taking the natural extension (see [Roh64]), the result follows.

Remark 4. The fact that the natural extension of $(X, T)$ in general contains more points, i.e., more backward orbits, than the natural extension of $(\hat{X}, \hat{T})$ was already observed by Buzzi [Buz97, Buz99. He shows that the set of points in the natural extension of $(X, T)$ that are not represented in the natural extension of the Markov extension carry no measure of positive entropy (or of entropy near the maximal entropy for fairly general higher dimensional systems).

\section{Bernoulli-LiKe PROPERTIES}

In this section we will discuss Bernoulli-like properties of the natural extension and how to transfer them from the natural extension to the original system and back. Let us first recall some definitions.

By a two-sided (resp. one-sided) Bernoulli shift we mean a shift space $\left(\mathcal{A}^{\mathbb{Z}}, \sigma\right)\left(\operatorname{resp} .\left(\mathcal{A}^{\mathbb{N} 0}, \sigma\right)\right)$ on a finite or countable alphabet $\mathcal{A}$ with left shift $\sigma$, and equipped with a stationary product measure based on a probability vector $\left(p_{1}, \ldots, p_{n}\right)$. An invertible dynamical system $(Y, \mathcal{C}, \nu, F)$ that is isomorphic to a Bernoulli shift is called Bernoulli itself.

If $(X, \mathcal{B}, \mu, T)$ is non-invertible, and isomorphic to a one-sided Bernoulli shift, then it is called one-sided Bernoulli itself. This is a much stronger property than the natural extension of $(X, \mathcal{B}, \mu, T)$ being isomorphic to a two-sided Bernoulli shift (cf. [BH09]); if the latter happens, the non-invertible system is called Bernoulli. It is a well-known result by Ornstein Orn70] (and [Smo72] and Orn71] for infinite alphabets) that entropy is a complete invariant for two-sided Bernoulli systems with positive or infinite entropy, but this is not true in general for the non-invertible case.

One theorem for which having a geometric version of the natural extension is useful is Theorem 3 from Sal73] by Saleski. To apply this theorem, we first show that the natural extension $F$ has an induced transformation that is Bernoulli. Consider one of the rugs $R_{u}, u \in \alpha$, and define the first return times for $z \in R_{u}$ under $F$ as

$$
\tau_{u}(z)=\inf \left\{n \geq 1: F^{n} z \in R_{u}\right\} .
$$

By the Poincaré Recurrence Theorem, $\tau_{u}(z)<\infty$ for $\nu$-a.e. $z \in R_{u}$. Define the induced map $F_{u}: R_{u} \rightarrow R_{u}$ by $F_{u} z=F^{\tau_{u}(z)} z$.

Theorem 3. Each map $F_{u}: R_{u} \rightarrow R_{u}, u \in \alpha$, is Bernoulli.

Proof. Consider the partition $\mathcal{P}=\left\{P_{1}, P_{2}, \ldots\right\}$ of $D_{u}$ into sets $P_{n}$ such that

$$
P_{n}=\left\{\hat{\pi}(z) \in D_{u}: \tau_{u}(z)=n\right\} .
$$

The map $\phi$ from Definition 1 acts as projection $\phi: R_{u} \rightarrow D_{u}$. For each $z \in R_{u}$ with $\tau_{u}(z)=n$, there is a corresponding $n$-path in $(\mathcal{D}, \rightarrow)$ from $D_{u}$ to $D_{u}$. Hence, there is an $(n+1)$-cylinder 
$Z_{j_{0} \cdots j_{n}}$, such that $\phi(z) \in Z_{j_{0} \cdots j_{n}}$ and $\hat{T}^{n}\left(\pi^{-1}\left(Z_{j_{0} \cdots j_{n}}\right) \cap D_{u}\right)=D_{u}$. Therefore, each set $P_{n}$ can be written as a finite union of pairwise disjoint sets:

$$
P_{n}=\bigcup\left\{\pi^{-1}\left(Z_{j_{0} \cdots j_{n}}\right) \cap D_{u}: Z_{j_{0} \cdots j_{n}} \in \mathcal{Z}_{n+1}, T^{n} Z_{j_{0} \cdots j_{n}}=\pi\left(D_{u}\right)\right\} .
$$

Note that the value of $\tau_{u}(z)$ for $z=(\hat{x}, y, u) \in R_{u}$ does not depend on $y$. Thus, we can write $\tau_{u}(z)=\tau_{u}(\hat{x})$ for almost all $\hat{x} \in D_{u}$. Define the map $\hat{T}_{u} \hat{x}=\hat{T}^{\tau_{u}(\hat{x})} \hat{x}$. Then $\hat{T}_{u}\left(\pi^{-1}\left(Z_{j_{0} \cdots j_{n}}\right) \cap\right.$ $\left.D_{u}\right)=D_{u}$ and thus $\hat{T}_{u}$ is Bernoulli.

Using the same arguments as before, we see that $\left(R_{u}, \mathcal{C} \cap R_{u}, \nu_{u}:=\left.\nu\right|_{R_{u}}, F_{u}\right)$ is the natural extension of $\left(D_{u}, \hat{\mathcal{B}} \cap D_{u},\left.\hat{\mu}\right|_{D_{u}}, \hat{T}_{u}\right)$ with factor map $\hat{\pi}$. Since $\hat{T}_{u}$ is Bernoulli, $F_{u}$ is Bernoulli as well.

Theorem 3 combined with Saleski's result implies the following.

Theorem 4 (Saleski [Sal73]). Suppose $(Y, \mathcal{C}, \nu, F)$ is weakly mixing. Fix $u \in \alpha$ and suppose that the following entropy condition holds:

$$
H_{\nu_{u}}\left(\vee_{k=1}^{\infty} \vee_{n=1}^{\infty} F_{u}^{k} Y_{n} \mid \vee_{i=1}^{\infty} F_{u}^{i} \mathcal{P}\right)<\infty,
$$

where $Y_{n}=\left\{R_{u}-\cup_{j=1}^{n} F^{-j} R_{u}, R_{u} \cap \cup_{j=1}^{n} F^{-j} R_{u}\right\}$ and $\mathcal{P}$ is a Bernoulli partition of $\left(R_{u}, F_{u}\right)$. Then $F$ is a Bernoulli automorphism and hence $T$ is Bernoulli as well.

Recall the construction of the Markov shift at the end of Section 2.2. The invertibility of $F$ allows us to associate to $F$ a two-sided countable state topological Markov shift and to use all the results available for this type of maps. To construct this Markov shift, first assign to a.e. $z \in Y$ a two-sided sequence $b(z)=\left(b_{k}\right)_{k \in \mathbb{Z}}$ by setting $b_{k}=u$ if $F^{k} z \in R_{u}$. Define the map $\psi: Y \rightarrow \alpha^{\mathbb{Z}}$ by $\psi(z)=b(z)$ and let $\Omega=\psi(Y)$. On $\Omega$, let $\mathcal{P}$ denote the product $\sigma$-algebra and let $\sigma$ be the left shift as usual. The Markov measure $m_{\underline{v}, P}$ is given by the probability vector $\underline{v}=\left(v_{u}\right)_{u \in \alpha}$ with entries $v_{u}=\hat{\mu}\left(D_{u}\right)$ and the (possibly) infinite probability matrix $P=\left(p_{t, u}\right)_{t, u \in \alpha}$ defined by

$$
p_{t, u}=\frac{\bar{\mu}\left(D_{t} \cap \hat{T}^{-1} D_{u}\right)}{\bar{\mu}\left(D_{t}\right)}
$$

Proposition 5. The systems $(Y, \mathcal{C}, \nu, F)$ and $\left(\Omega, \mathcal{P}, m_{\underline{v}}, \sigma\right)$ are isomorphic with isomorphism $\psi$, i.e., $\psi$ satisfies (ne2), (ne3) and (ne4) and is injective.

Proof. To show that $\psi$ is $\nu$-a.e. injective, note that $\psi(z)=\psi\left(z^{\prime}\right)$ implies that $F^{n} z, F^{n} z^{\prime} \in D_{u_{n}} \subseteq$ $Z_{j_{n}}$ for some sequence $\left(u_{n}\right) \in \alpha^{\mathbb{Z}}$. Since $F$ is expanding in the horizontal direction, this is only possible if $z=z^{\prime}$. It is immediate that $\psi$ is surjective and bi-measurable and that $\psi \circ F=\sigma \circ \psi$. Furthermore, it is straightforward to check that $\nu \circ \psi^{-1}=m_{\underline{v}}$. Hence, $\psi$ is a bi-measurable bijection that satisfies (ne2), (ne3) and (ne4) and is thus an isomorphism.

A (non-invertible) map $T$ is called exact on $(X, \mathcal{B}, \mu)$ if $\cap_{n=1}^{\infty} T^{-n} \mathcal{B}=\{\emptyset, X\}$. An invertible map $F$ is called a $K$-automorphism on $(Y, \mathcal{C}, \nu)$ if there is a sub- $\sigma$-algebra $\mathcal{C}_{0} \subseteq \mathcal{C}$ satisfying (i) $F^{-1} \mathcal{C}_{0} \subseteq \mathcal{C}_{0}$, (ii) $\cap_{n=1}^{\infty} F^{-n} \mathcal{C}_{0}=\{\emptyset, Y\}$ and (iii) the $\sigma$-algebra generated by $\cup_{n=1}^{\infty} F^{n} \mathcal{C}_{0}$ equals $\mathcal{C}$. An ergodic Markov shift is a $K$-automorphism if and only if it is strong mixing (see [Ito87] for example). A result from Rohlin [Roh64 says that a map is a $K$-automorphism if and only if it is the natural extension of an exact transformation. This gives the following corollary.

Corollary 2. If $(X, \mathcal{B}, \mu, T)$ satisfies (c1)-(c6) and $\mu$ is $T$-invariant and liftable, then $T$ is exact if and only if $F$ is a K-automorphism if and only if $F$ is strongly mixing if and only if the associated Markov shift $\left(\Omega, \mathcal{P}, m_{\underline{v}}, \sigma\right)$ is irreducible and aperiodic. 


\section{INTERVAL MAPS}

In this section we apply the construction of the natural extension to the specific case of piecewise linear expanding interval maps.

4.1. Piecewise linear interval maps. Let $T:[0,1] \rightarrow[0,1]$ be a piecewise linear expanding map. Let the partition $\mathcal{Z}$ consist of the closures of all maximal intervals of monotonicity for $T$. Then each set $D \in \mathcal{D}$ is an interval. Conditions (c1)-(c5) are immediate for Lebesgue measure $m$. Each set $Z_{j} \in \mathcal{Z}$ is an interval and thus $T^{n}\left(\cup_{j=1}^{N} \partial Z_{j}\right)$ consists of only finitely many points and $m\left(T^{n}\left(\cup_{j=1}^{N} \partial Z_{j}\right)\right)=0$. This implies (c6). Since $T$ is a piecewise linear expanding interval map Proposition 3 applies and $m$ is liftable. Hence the construction of the natural extension from Section 2.5 applies.

There always exists an ergodic invariant measure $\mu \ll m$ on $[0,1]$. This measure satisfies all conditions except possibly (c4). Recall the definition of capacity from (5). Note that $\#\left(\partial_{n} D\right)=2$ for all $n$ and hence $\operatorname{cap}(T)=0$. Then a $T$-invariant measure $\mu$ is liftable whenever $h_{\mu}(T)>0$. This was first proved by Hofbauer in [Hof79]. Hence, if one can show (c4) in a particular case, then one could also use the measure $\mu$.

This class of maps includes any piecewise linear expanding map of which the absolute value of the slope is constant. Here the entropy is equal to the log of the absolute value of the slope. For such maps, there is an additional result by Rychlik Ryc83: If the natural extension map $F$ is a $K$-automorphism, then $F$ is weakly Bernoulli, i.e., for each $\varepsilon>0$ there is a positive integer $N$, such that for all $m \geq 1$, all sets $A \in \bigvee_{k=0}^{m} F^{-k} \mathcal{C}$ and $C \in \bigvee_{k=-N-m}^{-N} F^{-k} \mathcal{C}$ we have

$$
|\nu(A \cap C)-\nu(A) \nu(C)|<\varepsilon,
$$

where $\bigvee_{i=\ell}^{k} F^{-i} \mathcal{C}$ denotes the smallest $\sigma$-algebra containing all elements in $F^{-i} \mathcal{C}$. The weak Bernoullicity of $F$ implies that of $T$.

4.2. Positive and negative slope $\beta$-transformations. Let $1<\beta<2$. The positive slope $\beta$-transformation is defined by $T_{\beta} x=\beta x(\bmod 1)$. It is a very well studied map with many interesting properties. It has a unique measure of maximal entropy $\mu_{1}$, equivalent to $m$, with entropy $h_{\mu_{1}}(T)=\log \beta$. Note that the Hofbauer tower gives a $T$-invariant measure $\hat{\mu} \circ \pi^{-1}$ by lifting $m$. By ergodic decomposition this measure is either equal to $\mu_{1}$ or not ergodic. In

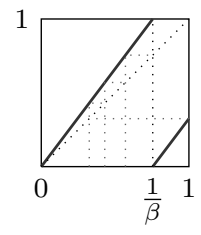

(a) The map $T$

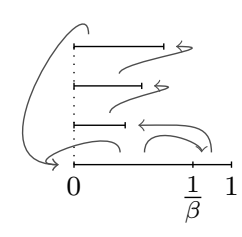

(b) The Hofbauer tower
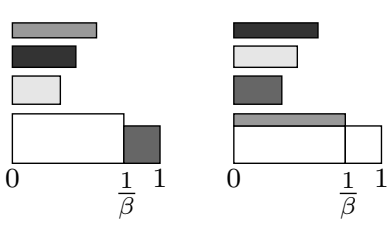

(c) The natural extension

FiguRE 5. In (a) we see the positive $\beta$-transformation with $\beta$ equal to the real root of $x^{3}-x-1$. (c) shows its natural extension. The transformation $F$ maps the areas on the left to the areas on the right with the same colour.

Figure 5 we see an example of a positive slope $\beta$-transformation for a specific value of $\beta$ and its natural extension. In [DKS96] Dajani et al. used a similar version of the geometric the natural extension of $T$ and showed that it is Bernoulli. Since all natural extensions of the same system are isomorphic ([Roh64] $)$, the natural extension given here is also Bernoulli.

Remark 5. Note that we start with Lebesgue measure $m$ on the unit interval. By Proposition 1 the construction produces an ergodic invariant probability measure $\hat{\mu} \circ \pi^{-1}$ for $T_{\beta}$. Since $\hat{\mu} \circ \pi^{-1} \ll$ $m$, and there is only one measure with these properties, we automatically have that $\hat{\mu} \circ \pi^{-1}=\mu_{1}$. 
The negative $\beta$-transformation is defined on the unit interval $[0,1]$ by $S x=-\beta x(\bmod 1)$. It has a unique measure of maximal entropy $\mu_{2}$, absolutely continuous with respect to Lebesgue. Also for this map $h_{\mu_{2}}(S)=\log \beta$.

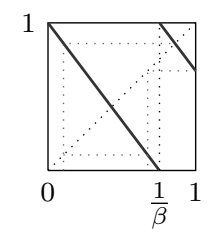

(a) The map $S$

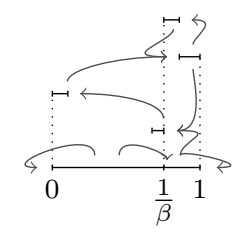

(b) The Hofbauer tower

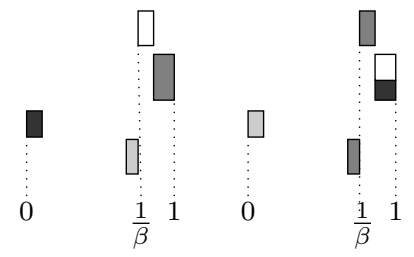

(c) The natural extension

FiguRE 6 . The negative $\beta$-transformation with $\beta$ equal to the real root of $x^{3}-$ $x-1$ and its natural extension.

Note that in general $\mu_{2}$ is not necessarily equivalent to Lebesgue on the unit interval, see Figure 6 for a specific example. In [LS11] Liao and Steiner show that $S$ is exact, hence by results from Rohlin [Roh64, the natural extension is a $K$-automorphism. Now the previously mentioned result from Rychlik [Ryc83] gives that the natural extension of $S$ is weakly Bernoulli and thus so is $S$ itself.

Now suppose that 1 has an eventually periodic orbit for both $T$ and $S$. This happens for example when $\beta$ is a Pisot number, i.e., a real-valued algebraic integer larger than 1 with all its Galois conjugates in modulus less than 1 (see Sch80 for $T$ and [FL09] for $S$ ). Then the natural extensions of both maps contain only finitely many rugs and the Markov shifts constructed in Section 2.5 have finite alphabets. Let $\left(Y_{T}, \mathcal{C}_{T}, \nu_{T}, F_{T}\right)$ and $\left(Y_{S}, \mathcal{C}_{S}, \nu_{S}, F_{S}\right)$ denote the natural extensions of $T$ and $S$. Since both $T$ and $S$ have the same entropy and are exact and thus strongly mixing, also $F_{T}$ and $F_{S}$ have the same entropy and are strongly mixing. The results from [KS79] by Keane and Smorodinsky show that then the natural extensions $T$ and $S$ are finitarily isomorphic, i.e., there is an a.e. continuous isomorphism from $Y_{T}$ to $Y_{S}$.

\section{Further exAmples}

5.1. Higher integer dimensions. Let $T:[0,1]^{d} \rightarrow[0,1]^{d}$ be a piecewise affine expanding map of the form $T x=A x\left(\bmod \mathbb{Z}^{d}\right)$. Let $Z_{1}, \ldots, Z_{N}$ be the pieces on which $T$ is continuous. Since $T$ is expanding in all directions, we get (c1) and (c3). The ergodicity from (c5) is clear for Lebesgure measure $m^{d}$ and since $T$ is piecewise affine, (c2) also holds. For each $1 \leq j \leq N$ and each measurable set $E \subseteq Z_{j}$ we have $m^{d}(T E)=|\operatorname{det}(A)| m^{d}(E)$, which gives (c4). Condition (c6) follows from the fact that $m^{d}\left(\cup_{j=1}^{N} \partial Z_{j}\right)=0$ combined with (c2) and (c4). Hence, $m^{d}$ satisfies conditions (c1)-(c6) and is not $T$-invariant. Then by Proposition $3, m^{d}$ is liftable and the construction from Section 2.5 gives the natural extension.

5.1.1. Random $\beta$-transformation. One specific example of a piecewise affine conformal map we give here is a variation of the random $\beta$-transformation, which was first introduced in [DK03. If $1<\beta<2$, then almost every point has infinitely many different number expansions of the form $\sum_{k=1}^{\infty} \frac{b_{k}}{\beta^{k}}$, where $b_{k} \in\{0,1\}$. The random $\beta$-transformation gives for each point all possible such expansions in base $\beta$ and is basically defined as the product of an independent coin tossing process and two isomorphic copies of the map $x \mapsto \beta x(\bmod 1)$ on an extended interval. Consider 
the space $X=[0,1] \times\left[0, \frac{1}{\beta-1}\right]$, with the partition $\mathcal{Z}=\left\{Z_{j}\right\}_{j=1}^{6}$ given by

$$
\begin{aligned}
& Z_{1}=\left[0, \frac{1}{2}\right) \times\left[0, \frac{1}{\beta}\right), \quad Z_{2}=\left[0, \frac{1}{2}\right) \times\left[\frac{1}{\beta}, \frac{1}{\beta(\beta-1)}\right], \quad Z_{3}=\left[0, \frac{1}{2}\right) \times\left(\frac{1}{\beta(\beta-1)}, \frac{1}{\beta-1}\right] . \\
& Z_{4}=\left[\frac{1}{2}, 1\right] \times\left[0, \frac{1}{\beta}\right), \quad Z_{5}=\left[\frac{1}{2}, 1\right] \times\left[\frac{1}{\beta}, \frac{1}{\beta(\beta-1)}\right], \quad Z_{6}=\left[\frac{1}{2}, 1\right] \times\left(\frac{1}{\beta(\beta-1)}, \frac{1}{\beta-1}\right] .
\end{aligned}
$$

The transformation $T: X \rightarrow X$ is defined by

$$
T(x, y)= \begin{cases}(2 x(\bmod 1), \beta y), & \text { if }(x, y) \in Z_{1} \cup Z_{2} \cup Z_{4}, \\ (2 x(\bmod 1), \beta y-1), & \text { if }(x, y) \in Z_{3} \cup Z_{5} \cup Z_{6} .\end{cases}
$$

The reasons why $(\mathrm{c} 1)-(\mathrm{c} 6)$ hold for $m^{d}$ are the same as in the previous example. Proposition 3 gives that $m^{d}$ is liftable to a measure $\hat{\mu}$ on the Hofbauer tower. Hence, we can construct the natural extension of $T$ as outlined in Section 2.5.

Originally the random $\beta$-transformation is not defined as a proper skew product, see [DK03]. Instead of always applying the doubling map in the second coordinate, they only apply the doubling map in the middle region. Below we give a specific example of the random $\beta$-transformation defined in this way and construct the natural extension for this value of $\beta$. Let $\beta=\frac{1+\sqrt{5}}{2}$ be the golden ratio and define the map $K:[0,1] \times[0, \beta] \rightarrow[0,1] \times[0, \beta]$ by

$$
K(x, y)= \begin{cases}(y, \beta x), & \text { if } x<1 / \beta, \\ (2 y, \beta x), & \text { if }(x, y) \in[1 / \beta, 1] \times[0,1 / 2), \\ (2 y-1, \beta x-1), & \text { if }(x, y) \in[1 / \beta, 1] \times[1 / 2,1] \\ (y, \beta x-1), & \text { if } x>1\end{cases}
$$

Then

$$
\begin{array}{ll}
Z_{1}=[0,1] \times[0,1 / \beta], & Z_{2}=[0,1 / 2] \times[1 / \beta, 1], \\
Z_{3}=[1 / 2,1] \times[1 / \beta, 1], & Z_{4}=[0,1] \times[1, \beta] .
\end{array}
$$

Since this already is a Markov partition, $\mathcal{D}=\left\{Z_{1}, Z_{2}, Z_{3}, Z_{4}\right\}$. Using the formula from (7) we get that for each $n \geq 2$,

$$
\rho_{n}\left(Z_{1}\right)=\frac{1}{n}\left(1+\sum_{k=2}^{n} \frac{f_{k+2}}{2 \beta^{k}}\right)
$$

where $f_{j}$ is the $j$-th element in the Fibonacci sequence starting with $f_{1}=1, f_{2}=1$. Using the direct formula for the elements in the Fibonacci sequence gives

$$
\rho\left(Z_{1}\right)=\lim _{n \rightarrow \infty} \rho_{n}\left(Z_{1}\right)=\frac{\beta^{3}}{2\left(\beta^{2}+1\right)} .
$$

By symmetry we get the same value for $Z_{4}$. Since $\rho\left(Z_{2}\right)=\frac{\rho\left(Z_{1}\right)}{\beta}+\frac{\rho\left(Z_{4}\right)}{\beta}$, we have $\rho\left(Z_{2}\right)=$ $\rho\left(Z_{3}\right)=\frac{\beta^{2}}{\beta^{2}+1}$. In Figure 7 we see the natural extension for this random $\beta$-transformation $K$.

5.2. Balanced measures. We say that $T: X \rightarrow X$ is $d$-to- 1 if there is a partition $\left\{Z_{j}\right\}_{j=1}^{d}$ of $X$, generating the $\sigma$-algebra of measurable sets, such that $T: Z_{j} \rightarrow X$ is a measurable bijection and $Z_{i} \cap Z_{j}$ is negligible (e.g. countable or of measure zero w.r.t. the measure used). A measure $\mu$ is balanced if the Jacobian $J(x) \equiv d$. In this case, $(\mathcal{D}, \rightarrow)$ is the full graph on $\left\{Z_{1}, \ldots, Z_{d}\right\}$, so conditions (c4) and (c6) are trivially satisfied. Therefore we can construct the natural extension by the method of Section 2.5 if the system satisfies (c1), (c2) and (c3) and if the measure is ergodic. We give two examples. 


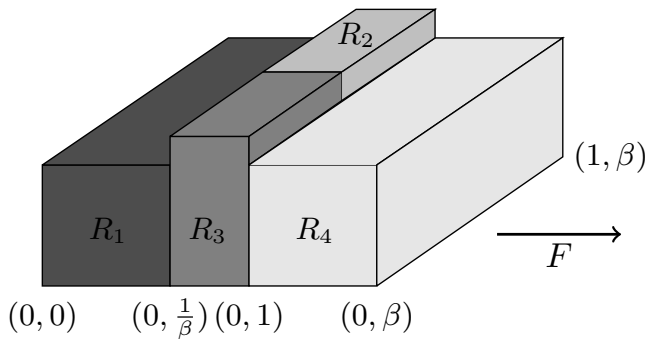

(a)

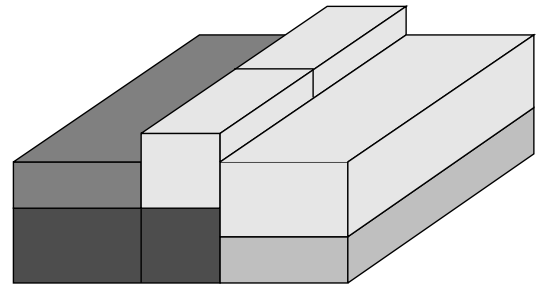

(b)

FiguRE 7 . The natural extension for the random $\beta$-transformation with $\beta$ equal to the golden ratio. $F$ maps the regions in (a) to the regions in (b) with the same colour.

5.2.1. Rational maps on the Julia set. Let $R: \hat{\mathbb{C}} \rightarrow \hat{\mathbb{C}}$ be a rational map of degree $d \geq 2$ on the Riemann sphere, i.e., $R(z)=\frac{P(z)}{Q(z)}$ where $P$ and $Q$ are two polynomials with no common factor and $d=\max \{\operatorname{deg} P, \operatorname{deg} Q\}$. When restricted to the Julia set, one can find a generating partition $\left\{Z_{j}\right\}$ w.r.t. which $R$ is $d$-to-1 giving (c1) and (c3). This goes back to Mañé [Mañ83] and the corresponding balanced measure is well-defined (i.e., independent of the choice of $\left\{Z_{j}\right\}$ ) as well as the unique invariant measure of maximal entropy. This gives (c2). Following conjectures and partial results by Mañé [Mañ85] and Lyubich [Lyu83], and using techniques of Hoffman and Rudolph [HR02] it was shown that $\mu$ is isomorphic to the $(1 / d, \ldots, 1 / d)$ one-sided Bernoulli shift, see [HH02], and hence $\mu$ is ergodic. Explicit construction for a Bernoulli partitions (for Lattès examples) can be found in [BK00, Kos02].

5.2.2. Certain endomorphisms on the torus. Let $X=\mathbb{T}^{n}=\mathbb{R}^{n} / \mathbb{Z}^{n}$ be the $n$-dimensional torus, and $T$ an endomorphism of the form $T(x)=h(A x)\left(\bmod \mathbb{Z}^{n}\right)$, where $h: \mathbb{T}^{n} \rightarrow \mathbb{T}^{n}$ is a homeomorphism homotopic to the identity, and $A$ an $n \times n$ integer matrix with $\operatorname{det}(A)= \pm d$. If $h$ is the identity, then Lebesgue measure is a balanced measure, see [DH93] for some intricancies of its natural extensions and factor spaces. A priori, a $d$-to- 1 partition $\left\{Z_{j}\right\}_{j=1}^{d}$ need not be unique; more importantly, it is not automatically generating. For example, if

$$
A=\left(\begin{array}{ll}
6 & 4 \\
2 & 2
\end{array}\right)=\left(\begin{array}{ll}
3 & 2 \\
1 & 1
\end{array}\right) \cdot\left(\begin{array}{ll}
2 & 0 \\
0 & 2
\end{array}\right)
$$

with eigenvalues $\lambda_{ \pm}=4 \pm 2 \sqrt{3}$, then the eigenspace of the second eigenvalue represents a contracting direction, and for this reason the partition of $\mathbb{T}^{2}$ in, say, four quarters, is not generating in forward time. In fact, there exists no forward time generating 4-to-1 partition because the topological entropy is $\log (4+2 \sqrt{3})>\log 4$. See Kowalski [Kow88] for some interesting results in this direction.

\section{REFERENCES}

[Aki02] S. Akiyama, On the boundary of self affine tilings generated by Pisot numbers, J. Math. Soc. Japan, 54(2):283-308 (2002).

[BK00] J. Barnes and L. Koss, One-sided Lebesgue Bernoulli maps of the sphere of degree $n^{2}$ and $2 n^{2}$, Int. J. Math. Math. Sci., 23(6):383-392 (2000).

[Bru95] H. Bruin, Combinatorics of the kneading map, In Proceedings of the Conference "Thirty Years after Sharkovskiı's Theorem: New Perspectives" (Murcia, 1994), 5:1339-1349 (1995).

[BH09] H. Bruin and J. Hawkins, Rigidity of smooth one-sided Bernoulli endomorphisms, New York Journal of Maths., 15:451-483 (2009).

[BT07] H. Bruin and M. Todd, Markov extensions and lifting measures for complex polynomials, Ergodic Theory Dynam. Systems, 27(3):743-768 (2007).

[BT09] H. Bruin and M. Todd, Equilibrium states for interval maps: the potential $-t \log |D f|$, Ann. Sci. Ec. Norm. Sup., 42(4):559-600 (2009). 
[Buz95] J. Buzzi, Entropies et représentations markoviennes des applications régulières de l'intervalle, $\mathrm{PhD}$ thesis, Orsay (1995).

[Buz97] J. Buzzi, Intrinsic ergodicity of smooth interval maps, Israel J. Math., 100:125-161 (1997).

[Buz99] J. Buzzi, Markov extensions for multi-dimensional dynamical systems, Israel J. Math., 112:357-380 (1999).

[DK03] K. Dajani and C. Kraaikamp, Random $\beta$-expansions, Ergodic Theory Dynam. Systems, 23(2):461-479 (2003).

[DH93] K. Dajani and J. Hawkins, Rohlin factors, product factors, and joinings for $n$-to-one maps, Indiana Univ. Math. J., 42(1):237-258 (1993).

[DK09] K. Dajani and C. Kalle., A natural extension for the greedy $\beta$-transformation with three arbitrary digits, Acta Math. Hungar., 125(1-2):21-45 (2009).

[DKS96] K. Dajani, C. Kraaikamp, and B. Solomyak, The natural extension of the $\beta$-transformation, Acta Math. Hungar., 73(1-2):97-109 (1996).

[FL09] C. Frougny and A. C. Lai, On negative bases, In Developments in language theory, Lecture Notes in Comput. Sci., 5583:252-263. Springer, Berlin (2009).

[Gur69] B. M. Gurevič, Topological entropy of a countable Markov chain, Dokl. Akad. Nauk SSSR, 187:715-718 (1969).

[Gur70] B. M. Gurevič, Shift entropy and Markov measures in the space of paths of a countable graph, Dokl. Akad. Nauk SSSR, 192:963-965 (1970).

[HH02] D. Heicklen and C. Hoffman, Rational maps are $d$-adic Bernoulli, Ann. of Math. (2), 156(1):103-114 (2002).

[Hof79] F. Hofbauer, On intrinsic ergodicity of piecewise monotonic transformations with positive entropy, Israel J. Math., 34(3):213-237 (1980).

[Hof80] F. Hofbauer, The topological entropy of the transformation $x \mapsto$ ax $(1-x)$, Monatsh. Math., 90(2):117141 (1980).

[HR89] F. Hofbauer and P. Raith, Topologically transitive subsets of piecewise monotonic maps which contain no periodic points, Monatsh. Math., 107(3):217-239 (1989).

[HR02] C. Hoffman and D. Rudolph, Uniform endomorphisms which are isomorphic to a Bernoulli shift, Ann. of Math. (2), 156(1):79-101 (2002).

[Ito87] K. Itō, Encyclopedic dictionary of mathematics. Vol. I-IV. MIT Press, Cambridge (MA) (1987).

[IR06] S. Ito and H. Rao, Atomic surfaces, tilings and coincidence. I. Irreducible case, Israel J. Math., 153:129$155(2006)$.

[Jag86] H. Jager, The distribution of certain sequences connected with the continued fraction, Nederl. Akad. Wetensch. Indag. Math., 48(1):61-69 (1986).

[JK89] H. Jager and C. Kraaikamp, On the approximation by continued fractions, Nederl. Akad. Wetensch. Indag. Math., 51(3):289-307 (1989).

[KH95] A. Katok and B. Hasselblatt, Introduction to the modern theory of dynamical systems. Cambridge University Press, Cambridge (1995).

[Kel89] G. Keller, Lifting measures to Markov extensions, Monatsh. Math., 108(2-3):183-200 (1989).

[Kel90] G. Keller, Exponents, attractors and Hopf decompositions for interval maps, Ergodic Theory Dynam. Systems, 10: 717-744 (1990).

[Kos02] L. Koss, Ergodic and Bernoulli properties of analytic maps of complex projective space, Trans. Amer. Math. Soc., 354(6):2417-2459 (2002).

[Kow88] Z. Kowalski, Minimal generators for ergodic endomorphisms, Studia Math., 91(2):85-88 (1988).

[KS79] M. Keane and M. Smorodinsky, Finitary isomorphisms of irreducible Markov shifts, Israel J. Math., 34(4):281-286 (1980).

[KS10] C. Kalle and W. Steiner, Beta-expansions, natural extensions and multiple tilings, Trans. Amer. Math. Soc., 364(5): 2281-2318 (2012).

[KSS10] C. Kraaikamp, T. Schmidt, and W. Steiner, Natural extensions and entropy of $\alpha$-continued fractsions, Nonlinearity, 25(8): 2207-2243 (2012).

[Lyu83] M. J. Lyubich, Entropy properties of rational endomorphisms of the Riemann sphere, Ergodic Theory Dynam. Systems, 3(3):351-385 (1983).

[LS11] L. Liao and W. Steiner, Dynamical properties of the negative beta-transformation, Ergodic Theory Dynam. Systems, 32(5):1673-1690 (2012).

[Mañ83] R. Mañé, On the uniqueness of the maximizing measure for rational maps, Bol. Soc. Brasil. Mat., 14(1):27-43 (1983).

[Mañ85] R. Mañé, On the Bernoulli property for rational maps, Ergodic Theory Dynam. Systems, 5(1):71-88 (1985).

[dMvS] W. de Melo and S. van Strien, One dimensional dynamics. Ergebnisse Series 25, Springer-Verlag (1993). 
[Orn70] D. Ornstein, Two Bernoulli shifts with infinite entropy are isomorphic, Advances in Mathematics, 5:339348 (1970).

[Orn71] D. Ornstein, Some new results in the Kolmogorov-Sinai theory of entropy and ergodic theory, Bull. Amer. Math. Soc., 77:878-890 (1971).

[Par69] W. Parry, Entropy and generators in ergodic theory. W. A. Benjamin, Inc., New York-Amsterdam (1969).

[PSZ08] Ya. B. Pesin, S. Senti and K. Zhang, Lifting measures to inducing schemes, Ergodic Theory Dynam. Systems, 28(2):553-574 (2008).

[Roh64] V. A. Rohlin, Exact endomorphism of a Lebesgue space, Magyar Tud. Akad. Mat. Fiz. Oszt. Közl., 14:443-474 (1964).

[Ryc83] M. Rychlik, Bounded variation and invariant measures, Studia Math., 76(1):69-80 (1983).

[Sal73] A. Saleski, On induced transformations of Bernoulli shifts, Math. Systems Theory, 7:83-96 (1973).

[Sal88] I. A. Salama, Topological entropy and recurrence of countable chains, Pacific J. Math., 134(2):325-341 (1988).

[Sch80] K. Schmidt, On periodic expansions of Pisot numbers and Salem numbers, Bull. London Math. Soc., 12(4):269-278 (1980).

[Sch00] K. Schmidt, Algebraic coding of expansive group automorphisms and two-sided beta-shifts, Monatsh. Math., 129(1):37-61 (2000).

[Smo72] M. Smorodinsky, On Ornstein's isomorphism theorem for Bernoulli shifts, Advances in Math., 9: 1-9 (1972).

[Tsu01] M. Tsujii, Absolutely continuous invariant measures for expanding linear maps, Invent. Math., 143:(2):349-373 (2001).

[Wal82] P. Walters, An introduction to ergodic theory. Springer Verlag (1982).

Henk Bruin

Fakultät für Mathematik

Universität Wien

Nordbergstraße 15/Oskar Morgensternplatz 1, A-1090 Wien

Austria

henk.bruin@univie.ac.at

Charlene Kalle

Mathematisch Instituut

Leiden University

Niels Bohrweg 1, 2333CA Leiden

The Netherlands

kallecccj@math.leidenuniv.nl 